\title{
Envelhecimento, obesidade e consumo alimentar em idosos
}

\author{
Aging, obesity and food consumption in the elderly
}

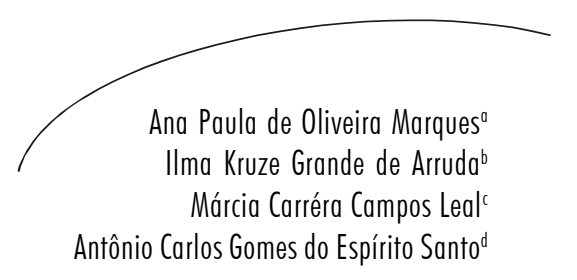

\section{RESUMO}

Com o envelhecimento da população predominam as enfermidades crônicas e, nesse conjunto, destaca-se a obesidade, por sua prevalência sempre crescente nos diversos grupos etários, incluindo os idosos, e por suas complicações, quer de forma isolada ou associada a outros eventos mórbidos, como: hipertensão arterial, diabetes mellitus não-insulino dependente e doenças cardiovasculares, enfermidades com prevalências já elevadas entre pessoas idosas. A obesidade, considerada um problema de saúde pública, representa para o idoso um risco adi cional de importância considerável. Apesar dos múltiplos fatores que determinam o consumo alimentar, aspectos relacionados à adoção de dietas com elevado valor energético, ricas em gorduras de origem animal, açúcares, alimentos refinados e com reduzido teor de frutas, verduras e fibras, sugerem contribuição favorável à ocorrência da obesidade.

\author{
Palavras-chave: \\ envelhecimento; \\ doenças; obesidade; \\ prevalência; saúde \\ pública
}

\section{ABSTRACT}

People are growing older, and chronic diseases are becoming predominant; in this scenery obesity stands out, due to its increasing prevalence among the several age groups, including the elderly, and for its complications, either alone or associated with other morbid events, such as: arterial hypertension, diabetes mellitus non-insulin dependent and cardiovascular diseases, which have high prevalence among aged people. Obesity, considered a public health problem, is an additional important risk for the elderly. Despite the multiple factors that

Correspondência / Correspondence

Ana Paula de Oliveira Marques

Rua João Eugênio de Lima, 67/402 - Boa Viagem

51020-630 - Recife, PE, Brasil

E-mail: marquesap@ hotmail.com 
determine alimentary consumption, aspects related to highly energetic diets, rich in animal fat, sugars, refined foods, and less fruits, vegetables and fibers content, suggest a contribution that favours que occurrence of obesity.
Key words: aging; disease; obesity; prevallence; public health

\section{INTRODUÇÃO}

A Gerontologia tem por campo de investigação a descrição e a explicação das mudanças típicas do processo de envelhecimento e de seus determinantes biológicos, psicológicos e socioculturais. O grande desafio que se coloca está na consolidação de conhecimentos gerontológicos sob a ótica da interdisciplinaridade. Os idosos, a velhice e o envelhecimento representam o foco central de interesse. ${ }^{26}$

Apesar de sua aplicabilidade, o critério etário não é suficiente para explicar o envelhecimento, considerando a variabilidade com que cada indivíduo se comporta neste domínio. A influência de fatores ambientais, genéticos e comportamentais contribui para explicar a desigualdade do envelhecer, ressaltando a importância da idade biológica em relação à cronológica. ${ }^{11}$

A larga utilização do critério cronológico em trabalhos científicos justifica-se pela dificuldade de definição da idade biológica, tendo como ponto de partida as visões contraditórias sobre o início do processo de envelhecimento e a inexistência de marcadores biofisiológicos eficazes e confiáveis na mensuração do envelhecimento, dificultando a comparabilidade de dados. ${ }^{26}$

O grande desafio deste século será cuidar de uma população envelhecida, em sua mai- oria com níveis socioeconômicos e educacionais baixos e com alta prevalência de doenças crônicas e incapacitantes. ${ }^{29}$

No cuidado com o segmento mais envelhecido, vale destacar a urgência na reestruturação dos espaços sociais no acolhimento adequado à pessoa idosa, incluindo os serviços de saúde e a formação de profissionais devidamente qualificados para promoção, proteção e recuperação da saúde daqueles que, superando as adversidades ao longo da vida, conseguiram envelhecer.

\section{Envelhecimento, obesidade e co-morbidade}

A prevalência de obesidade vem aumentando nas últimas décadas, em todas as faixas de idade, tendo comportamento semelhante ao envelhecimento populacional enquanto fenômeno mundial.

A obesidade, conceituada como o excesso de tecido adiposo no organismo, representa atualmente um problema de saúde pública, em função de sua magnitude e pela relação direta ou indireta com outras enfermidades ou situações patológicas de importância considerável. ${ }^{5,31}$

Para classificação do estado nutricional de adultos e idosos a partir da utilização do Índice de Massa Corporal - IMC, que consiste 
na divisão do peso corporal, em gramas, pela altura, em metros quadrados, a OMS propôs a substituição da terminologia sobrepeso, adotada na recomendação anterior ${ }^{23}$ para obesidade, a partir do IMC $\geq 30 \mathrm{Kg} / \mathrm{m}^{2}$, e a inclusão de uma subdivisão adicional. Os valores de IMC situados entre 25,0 - 29,9 Kg/ ${ }^{2}$, referidos anteriormente como sobrepeso grau I, na nova recomendação correspondem à condição de pré-obesidade. ${ }^{34}$

Quadro 1 - Classificação de Adultos segundo o Índice de Massa Corporal - IMC.

\begin{tabular}{|lc|}
\hline Classificação & $\mathrm{IMC}\left(\mathrm{Kg} / \mathrm{m}^{2}\right)$ \\
\hline Abaixo do peso & $<18,5$ \\
Faixa normal & $18,5-24,9$ \\
Sobrepeso & $\geq 25$ \\
Pré-obesidade & $25,0-29,9$ \\
Obesidade grau I & $30,0-34,9$ \\
Obesidade grau II & $35,0-39,9$ \\
Obesidade grau III & $\geq 40$ \\
\hline
\end{tabular}

Fonte: WHO, 1998.

Quanto à localização predominante do tecido adiposo e os riscos associados à saúde, a obesidade pode ser classificada em andróide e ginóide. $\mathrm{Na}$ forma de distribuição andróide, também chamada de obesidade abdominal ou central, o excesso de tecido adiposo predomina na metade superior do corpo, acima do umbigo, à frente e acima das vértebras L4 e L5. Essa forma em geral está associada à ocorrência de distúrbios metabólicos e doenças cardiovasculares, sendo mais predominante nos homens e em mulheres após a menopausa. A ocorrência simultânea de obesidade abdominal, dislipidemia, hipertensão arterial e diabetes, conhecida como síndrome metabólica ou síndrome de resistência à insulina, constitui fator de risco considerável para a ocorrência de doença cardiovascular.

$\mathrm{Na}$ forma de distribuição ginóide, o tecido adiposo predomina na metade inferior do corpo, apresenta menor repercussão metabólica, embora acarrete maiores conseqüências mecânicas, sendo mais prevalente em mulheres antes da menopausa. ${ }^{20}$

O excesso de peso corporal favorece a ocorrência de diabetes mellitus não-insulino dependente (DMNID), ou tipo 2, doenças cardiovasculares, hipertensão arterial, doenças da vesícula biliar e algumas formas de câncer. Outros prejuízos à saúde, mais prevalentes em indivíduos com sobrepeso, incluem: dificuldades respiratórias (apnéia do sono), problemas dermatológicos, distúrbios do aparelho locomotor, além da própria estigmatização social. ${ }^{32}$

Em relação ao estigma social, Sichieri ${ }^{31}$ refere que a obesidade, na sociedade contemporânea, não significa apenas o homem doente, mas também o excluído no imaginário popular, de uma estética socializada. Vale referir que o próprio envelhecimento representa um marcador de importância considerável para o aumento da prevalência de sobrepeso e co-morbidade associada.

O diabetes não-insulino dependente, ou tipo 2, é mais comum entre pessoas idosas, estando a condição em geral associada à obe- 
sidade, hipertensão arterial e a uma maior freqüência de complicações macrovasculares. Registra-se, para a população brasileira entre 60 e 69 anos, uma prevalência de diabetes de $17,4 \%, 6,4$ vezes superior à observada para 0 segmento etário de 30 a 39 anos. A glicemia de jejum aumenta de 1 a $2 \mathrm{mg}$ por decilitro, e a pós-prandial, de 8 a $20 \mathrm{mg}$ por década, após os 30-40 anos de idade. ${ }^{25}$

A associação entre ganho de peso e diabetes encontra-se descrita na literatura especializada. Em indivíduos com ganho de peso máximo de 10 a $20 \%$, a prevalência de diabetes correspondeu a $4 \%$; quando o ganho de peso se situou em torno de 40 a $50 \%$, esta prevalência aumentou para $20 \%$. Ganhos superiores a $50 \%$ representaram uma ocorrência de diabetes de quase $30 \%{ }^{10}$

Estudos prospectivos demonstram que a obesidade generalizada e, de forma particular, a obesidade andróide, favorecem o risco de desenvolvimento de diabetes tipo 2 , por induzirem maior resistência à insulina em sua ação de estimular a captação de glicose periférica. A redução de peso em indivíduos obesos diminui a resistência à insulina e o risco de desenvolvimento de diabetes. Em pacientes com a doença já instalada, a redução de peso melhora o controle glicêmico. ${ }^{3}$

Estima-se que a prevalência de diabetes deverá triplicar na faixa etária de 45 a 64 anos e duplicar no segmento etário dos maiores de 65 anos. O aumento das taxas de sobrepeso e obesidade, associado ao envelhecimento populacional e às alterações no estilo de vida, são os principais fatores que explicam a ten- dência de crescimento da prevalência de diabetes tipo 2 , observada nas últimas décadas, estando o risco diretamente associado ao aumento do Índice de Massa Corporal. ${ }^{30}$

A relação entre o excesso de adiposidade e a ocorrência de doença cardiovascular foi destacada no estudo de Framingham, em 26 anos de acompanhamento de uma casuística composta por 5.206 homens e mulheres com idade entre 35 e 84 anos. Para os homens, o peso teve valor preditivo em relação à incidência de doença coronariana e morte decorrente desta patologia e de insuficiência cardíaca, independentemente de idade, níveis de colesterol, pressão arterial sistólica, tabagismo, hipertrofia do ventrículo esquerdo e intolerância à glicose. Nas mulheres, o peso esteve associado positivamente e de forma independente à ocorrência de doença coronariana, acidente vascular cerebral e insuficiência cardíaca. De certa forma, os achados contribuíram para que fosse revisto o conceito benigno atribuído até então à obesidade, na ausência de fatores de risco para doença cardiovascular. ${ }^{3}$

Embora os achados sinalizem a obesidade como fator de risco independente para a doença cardíaca, é comum a ocorrência de dislipidemia, hipertensão arterial e intolerância à glicose em indivíduos com excesso de adiposidade, principalmente do tipo andróide, e com conseqüências importantes à integridade do sistema vascular.

Quanto ao impacto da obesidade no perfil de mortalidade, verifica-se grande variabilidade entre diferentes estudos; contudo, o padrão de distribuição mais central e o ganho 
de peso na fase adulta parecem induzir a aceleração do processo aterogênico.

As doenças cardiovasculares representam a principal causa de óbito na população idosa, e a hipertensão arterial situa-se nesse grupo como a mais prevalente, cujo risco aumenta com a evolução da idade. A combinação de sobrepeso e hipertensão arterial leva ao espessamento da parede ventricular e maior volume cardíaco, bem como aumenta a probabilidade de insuficiência cardíaca.

Resultados de estudos epidemiológicos sugerem, para fins de definição e tratamento da hipertensão arterial em idosos, pressão arterial sistólica (PAS) $\geq 140 \mathrm{mmHg}$ e/ou diastólica $(\mathrm{PAD}) \geq 90 \mathrm{~mm} \mathrm{Hg}$, valores idênticos aos adotados para indivíduos a partir de 18 anos. ${ }^{4}$

Nos idosos, a prevalência de hipertensão arterial sistólica isolada (HASI), definida a partir de valores $\mathrm{PAS} \geq 140 \mathrm{mmHg}$ e $\mathrm{PAD}<90$ $\mathrm{mmHg}$, assume importância considerável, presente em mais de dois terços dos hipertensos com idade entre 65 e 89 anos, e está associada ao aumento do risco de acidente vascular cerebral (AVC) e infarto agudo do miocárdio. ${ }^{8}$

Indivíduos obesos, sobretudo os portadores de obesidade abdominal, apresentam maior produção de ácidos graxos livres e, em geral, altas taxas de colesterol total, LDL-colesterol e triglicerídeos, e níveis de HDL-colesterol reduzidos. Estas modificações no LDL e HDL colesterol produzem aumento na relação LDL/HDL, elevando o risco aterogênico. O fluxo hepático maior de ácidos gra- xos livres leva a uma diminuição da ligação e extração de insulina pelo fígado, favorecendo a hiperinsulinemia, além de constituir substrato importante para a maior produção hepática de lipoproteínas de muito baixa densidade (VLDL), ricas em triglicerídeos. ${ }^{14}$

As dislipidemias representam fator de risco para doença coronariana em indivíduos idosos, apesar de esse risco diminuir com a idade. A hipercolesterolemia (colesterol total $\geq 240 \mathrm{mg} / \mathrm{dL}$ ) em pessoas idosas é mais prevalente nas mulheres do que nos homens, sendo mais freqüente na faixa dos 65 a 74 anos, diminuindo gradativamente após essa idade. ${ }^{21}$

Como valores desejáveis e de menor risco à saúde de indivíduos maiores de 20 anos, foram definidos: colesterol total $<200 \mathrm{mg} / \mathrm{dL}$, LDLcolesterol $<130 \mathrm{mg} / \mathrm{dL}$, HDL-colesterol $>35$ $\mathrm{mg} / \mathrm{dL}$ e triglicerídeos $<200 \mathrm{mg} / \mathrm{dL}^{?}$

\section{Prevalência de obesidade em idosos e fatores associados}

A associação entre fatores de risco cardiovascular e obesidade foi estudada em idosos finlandeses não-diabéticos (396 homens e 673 mulheres), com idades entre 65 e 74 anos. Os idosos com IMC $>27 \mathrm{Kg} / \mathrm{m}^{2}$ apresentaram níveis mais elevados de glicemia, triglicerídeos e pressão arterial sistólica e mais baixos de HDL-colesterol, quando comparados aos idosos com IMC $<27 \mathrm{Kg} / \mathrm{m}^{2}{ }^{23}$

A relação entre obesidade e hipertensão arterial foi determinada em 1.378 idosos (60 
a 82 anos), controlando-se as variáveis idade, atividade física, ingesta de álcool, tabagismo e diabetes mellitus. Os resultados descrevem redução dos valores de IMC com o aumento da idade e associação significativa entre obesidade e valores elevados de pressão arterial sistólica e diastólica. ${ }^{18}$

Investigação realizada no município do Rio de Janeiro, envolvendo 4.031 indivíduos, dentre os quais 631 idosos, revelou prevalência de sobrepeso (IMC $\geq 25 \mathrm{Kg} / \mathrm{m}^{2}$ ) no grupo mais envelhecido, equivalente a $46,9 \%$ e $54,6 \%$, para homens e mulheres, respectivamente. No tocante à escolaridade, agrupada em dois estratos (baixa e média; alta), as menores taxas de excesso de peso foram registradas entre as mulheres do segundo grupo. Quanto à renda, foi observado que o sobrepeso não era privilégio dos grupos mais favorecidos; pelo contrário, maiores prevalências foram registradas nos segmentos de menor renda. ${ }^{30}$

Em amostra composta por 586 indivíduos maiores de 65 anos, residentes numa comunidade tailandesa, sendo $66 \%$ homens e $34 \%$ mulheres, a prevalência de pré-obesidade $\left(25,0 \leq \mathrm{IMC}<30,0 \mathrm{Kg} / \mathrm{m}^{2}\right)$ e obesidade (IMC $\geq 30 \mathrm{Kg} / \mathrm{m}^{2}$ ) correspondeu a $24,8 \% \mathrm{e}$ $12,7 \%$ nos homens, e $29,7 \%$ e $11,5 \%$ nas mulheres, respectivamente. As duas condições se mostraram associadas, após o controle dos fatores de confusão, a hipertensão arterial, hipertrigliceridemia e hiperglicemia. ${ }^{13}$

Lerman et al., ${ }^{12}$ investigando a prevalência de obesidade (IMC $\geq 30 \mathrm{Kg} / \mathrm{m}^{2}$ ) e a associação com variáveis sociodemográficas, histó- ria médica, funcional e dados bioquímicos, em dois grupos de indivíduos - idosos (334) e não-idosos (273), com idades entre 35 e 59 anos, residentes em áreas urbanas e rurais do México -, identificaram uma prevalência de $23,6 \%$ de obesidade no grupo de homens mais jovens e 15,6\% entre os idosos. Nas mulheres, a prevalência de obesidade correspondeu a $28,4 \%$ e 19,7\%, para as não-idosas e idosas, respectivamente. A obesidade, nos idosos, se mostrou associada à hiperinsulinemia e à moradia em área urbana. No grupo dos mais jovens, a associação foi positiva para hiperinsulinemia, hipertensão arterial e distribuição mais central da adiposidade.

A situação nutricional da população idosa brasileira foi analisada segundo variáveis sociodemográficas, com amostra representativa composta por $2.028(47,4 \%)$ homens e 2.249 $(52,6 \%)$ mulheres. Entre os homens, a prevalência geral de magreza (IMC $\left.<18,5 \mathrm{Kg} / \mathrm{m}^{2}\right)$ correspondeu a 7,8\%, superior à de sobrepeso II e III (IMC $\geq 30 \mathrm{Kg} / \mathrm{m}^{2}$ ), equivalente a 5,7\%. Nas idosas, a tendência foi inversa: 8,4\% estavam na condição de magreza e 18,2\% apresentavam sobrepeso II e III. Quando comparados aos adultos na faixa etária de 20 a 40 anos, os homens idosos apresentaram freqüência de sobrepeso 1,5 vezes maior, enquanto nas idosas a freqüência foi 2,5 vezes superior. O sobrepeso foi mais freqüente em áreas urbanas das regiões Sul e Sudeste, nos grupos de maior renda, maior escolaridade e melhor qualidade de moradia. ${ }^{33}$

A prevalência de obesidade (IMC $\geq 30$ $\mathrm{Kg} / \mathrm{m}^{2}$ ), registrada em 847 idosos assistidos em unidades ambulatoriais, localizadas no 
município de Londrina-PR, foi significativamente maior nas mulheres $(23,8 \%)$ em relação aos homens $(9,6 \%)$, e proporcionalmente menor nos idosos mais velhos (80 anos e mais). Nos homens obesos, esteve associada a uma freqüência maior de diabetes, HDLcolesterol baixo e hipertrigliceridemia, em relação aos não-obesos (IMC $<30 \mathrm{Kg} / \mathrm{m}^{2}$ ). Nas idosas, a obesidade esteve associada apenas em relação à hipertensão arterial. ${ }^{6}$

Barreto, Passos e Lima Costa ${ }^{1}$, investigando a coexistência de obesidade e baixo-peso em uma casuística composta por 1.451 idosos residentes em Bambuí-MG, identificaram uma prevalência de obesidade de $12,5 \%$, diretamente associada ao sexo feminino, à maior renda familiar e à ocorrência de hipertensão arterial e diabetes, e inversamente associada à atividade física. O baixo-peso ocorreu em 14,8\% dos idosos, aumentando com a idade, sendo mais freqüente nos homens e nas famílias de menor renda, positivamente associado à infecção por Tripanossoma cruzi i a duas ou mais internações no último ano, anterior ao estudo, e inversamente à presença de hipertensão e diabetes.

Marques et al. ${ }^{16}$ analisando a prevalência de obesidade (IMC $\geq 30 \mathrm{Kg} / \mathrm{m}^{2}$ ) e fatores associados, em 188 mulheres idosas, com idade entre 60 e 89 anos, assistidas em unidade geronto-geriátrica, registraram uma prevalência de obesidade equivalente a 25,6\%. Esta esteve concentrada nos intervalos etários de 60 a 69 $\operatorname{anos}(28,8 \%)$ e 70 a 79 anos $(24,6 \%)$ e positivamente associada à glicemia de jejum e" 126 $\mathrm{mg} / \mathrm{dL}$, com risco maior para as idosas diabéticas, em relação às não-diabéticas. A maior probabilidade de ocorrência de obesidade, em torno de $18 \%$, foi observada nas mulheres com menos de 70 anos, triglicerídeos $\geq 200 \mathrm{~m} / \mathrm{dL}$, diabéticas e hipertensas, o que destaca a importância da morbidade associada.

\section{Consumo alimentar e envelhecimento}

Em paralelo às modificações ocorridas no perfil demográfico e epidemiológico da população brasileira, as mudanças verificadas no cenário nutricional registram declínio acentuado da desnutrição e aumento significativo da prevalência de pré-obesidade e obesidade, característica marcante da transição nutricional brasileira.

Segundo Popkin ${ }^{27}$, a transição nutricional diz respeito a alterações nos padrões nutricionais, como conseqüência de modificações na estrutura da dieta dos indivíduos, associadas a mudanças econômicas, sociais, demográficas e relacionadas à saúde.

Dietas com alta densidade energética, associadas a um estilo de vida sedentário, destacamse como fatores determinantes responsáveis pelo aumento da prevalência da obesidade, identificado em diversas partes do mundo. ${ }^{34}$

O consumo de uma dieta rica em gorduras, destacando-se as de origem animal, açúcar e alimentos refinados, reduzida em carboidratos complexos e fibras, denominada dieta ocidental, está positivamente associado à maior ocorrência de doenças cardiovasculares e obesidade.

No Brasil, Monteiro, Mondini e Costa ${ }^{19}$ verificaram um aumento no consumo de car- 
nes, leite, gorduras saturadas, açúcares e refrigerantes, em regiões metropolitanas, e redução na ingestão de cereais, frutas, verduras e legumes.

São múltiplos os fatores intervenientes no consumo alimentar, dentre os quais os fisiológicos, sociais, culturais, econômicos e os relacionados às condições de saúde. Nesse aspecto, a maior ocorrência de enfermidades, com o passar dos anos, representa, para a pessoa idosa, fator adicional de importância considerável na elaboração de estratégias de orientação nutricional direcionadas ao segmento idoso.

No Brasil, a insuficiência financeira da grande maioria da população idosa, dependente de aposentadorias e/ ou pensões, por vezes comprometidas com a aquisição de medicamentos, favorece a monotonia alimentar e a aquisição de alimentos de menor custo, ficando em segundo plano o valor nutricional. ${ }^{5}$

$\mathrm{Na}$ literatura especializada, os estudos sobre consumo alimentar em sua maioria estão focalizados na análise dos nutrientes. Sichier ${ }^{31}$ considera fundamentais as abordagens que levem em conta os alimentos e não só os nutrientes, porque as pessoas consomem os alimentos e não os nutrientes, especificamente. A autora ainda refere que recomendações nutricionais baseadas em alimentos são mais facilmente transformadas em práticas de saúde pública.

A seguir serão descritos estudos de base qualitativa, que incluem o segmento idoso e cujo objeto de análise diz respeito ao consumo de alimentos.
A dieta habitual, de acordo com a participação por grupo alimentar, foi identificada em uma casuística composta por 308 idosos, atendidos no Serviço de Geriatria do Hospital das Clínicas da Faculdade de Medicina da Universidade de São Paulo, no período entre abril de 1989 e junho de 1990. O pão, o café e o açúcar fizeram parte da dieta de todos os indivíduos, desde aquele que fazia apenas duas refeições diárias, até o que consumia mais de seis.

No grupo dos alimentos construtores, o consumo de carnes foi mais citado, ocupando as duas primeiras posições a bovina e de aves, respectivamente. No grupo dos alimentos energéticos, os cereais e derivados foram os mais referidos, destacando-se o consumo de arroz e pão, presentes na dieta de todos os idosos. Entre os alimentos reguladores, os legumes foram mais referidos em relação às verduras. A autora atribui o fato ao consumo de sopas, mais freqüente entre pessoas idosas, seja por questões de natureza econômica, ajuste à capacidade mastigatória ou facilidade de preparo. ${ }^{17}$

Najas et al., ${ }^{24}$ analisando o padrão de consumo alimentar de 283 idosos, sendo 46\% do sexo masculino e $54 \%$ do sexo feminino, agrupados em três regiões, por ordem crescente de nível socioeconômico, verificaram que, do grupo dos alimentos energéticos, o pão e o arroz foram os mais citados, com diferenciação de consumo por região. Enquanto o arroz foi menos consumido na região III, o pão o foi na região I, de menor poder aquisitivo. Em relação aos alimentos protéicos, apenas o feijão e o leite e derivados se destacaram em termos de consumo diário, 
sendo o leite e derivados os mais consumidos na região III. No grupo dos alimentos reguladores, as verduras e legumes foram pouco utilizados nas três regiões analisadas e o consumo diário de frutas não foi suficiente na região I.

O perfil antropométrico e dietético de 113 idosos, com idades entre 60 e 82 anos, participantes do Programa de Reabilitação Cardíaca e da Universidade Aberta à Terceira Idade (UnATI) da Universidade do Estado do Rio de Janeiro, foi investigado, sendo registrados elevados índices de sobrepeso e obesidade em ambos os sexos. O consumo diário de alimentos foi pesquisado mediante a aplicação de um questionário de freqüência alimentar, verificando-se que os mais consumidos foram leite, arroz, carne de frango, cenoura e laranja, sendo também expressiva a freqüência diária de café e óleo vegetal. ${ }^{9}$

O consumo de produtos industrializados e de fácil preparo, como doces, massas, biscoitos, bolachas, entre outros, é freqüente em circunstâncias domésticas desfavoráveis, como isolamento e solidão, situações comuns na velhice, e entre os idosos que apresentam algum comprometimento funcional, incluindo os cuidados com a alimentação, interferindo na nutrição. ${ }^{22}$

Pesquisa realizada por Sena, ${ }^{32} \operatorname{com} 117$ indivíduos participantes de grupos de convivência de idosos de João Pessoa-PB, avaliou a composição corporal e os fatores associados, incluindo hábitos alimentares, condições socioeconômicas e morbidade referida. Identificou-se um consumo qualitativo inadequado, em relação ao leite integral $(42,8 \%)$, carne vermelha $(85,5 \%)$, queijos gordurosos (67,5\%), manteiga, salsicha, salame e toucinho $(59,9 \%)$, ovos $(73,5 \%)$ e carboidratos simples $(81,2 \%)$. O autor destaca a necessidade de orientação nutricional, principalmente em função da morbidade (hipertensão, diabetes, hipercolesterolemia) associada à condição de sobrepeso, cuja prevalência correspondeu a 70,2\% para as mulheres e $53,8 \%$ para os homens.

O consumo alimentar de 126 idosos, alunos de cursos da UnATI / UFPE, foi investigado por Bion ${ }^{2}$ et al. (2003), mediante recordatório de 24h. Os alimentos consumidos com maior freqüência corresponderam às frutas $(94,5 \%)$, vegetais $(85,3 \%)$, leite e derivados $(84,4 \%)$. Percentual idêntico de consumo foi identificado para as leguminosas, doces e açúcares, referidos por $41,3 \%$ da casuística.

Marques et al., ${ }^{16}$ analisando a freqüência de consumo alimentar em 100 mulheres idosas, incluindo os dez alimentos mais citados e suas freqüências diária, semanal e mensal por grupo alimentar, verificaram que o arroz e o pão estavam entre os alimentos mais referidos em termos de consumo diário no grupo dos energéticos. Em relação à freqüência semanal, o feijão, o bolo, e a farinha de mandioca foram os mais referidos. O leite desnatado e o leite integral ocuparam as duas primeiras posições no grupo dos alimentos construtores, e as carnes de frango e bovina foram mais citadas em termos de freqüência semanal. $\mathrm{O}$ grupo dos alimentos reguladores foi o mais comprometido, com reduzido consumo de frutas e vegetais. 


\section{CONSIDERAÇÕES FINAIS}

Como os idosos representam atualmente o segmento populacional que mais cresce em termos proporcionais, ressalta-se a importância das questões relacionadas ao envelhecimento e à velhice. Concomitantemente à transição epidemiológica, marcada pela predominância de enfermidades crônicas, típicas de populações mais envelhecidas, situa-se a transição nutricional, marcada pelo aumento na prevalência da obesidade, com repercussões clínicas e sociais importantes, principalmente entre os maiores de 60 anos.

Evidências na literatura especializada destacam a associação entre o consumo alimentar e a ocorrência de enfermidades crônicas, ressaltando a importância da nutrição como ferramenta para prevenção e controle dessas doenças.

\section{NOTA}

\footnotetext{
Doutora em Nutrição pela UFPE, Professora Adjunta no Departamento de Medicina Social da Universidade Federal de Pernambuco. Especialista em Gerontologia Social pela UPE. Coordenadora do Programa do Idoso - PROIDOSO, da Universidade Federal de Pernambuco, Recife, PE, Brasil.

b Doutora em Nutrição pela UFPE, Professora Adjunta no Departamento de Nutrição da Universidade Federal de Pernambuco, Recife, PE, Brasil.

c Doutora em Odontologia Social pela UPE, Professora Adjunta no Departamento de Medicina Social da Universidade Federal de Pernambuco, Coordenadora do Núcleo de Atenção ao Idoso - NAI, Universidade Federal de Pernambuco, Recife, PE, Brasil.

d Doutor em Saúde Pública pela USP, Professor Adjunto no Departamento de Medicina Social da Universidade Federal de Pernambuco - UFPE, Recife, PE, Brasil.
}

\section{REFERÊNCIAS}

1. Barreto SM, Passos VMA, Lima Costa MF. Obesity and underweight among Brazilian elderly. The Bambuí health and aging study. Cad Saúde Pública 2003 [acesso 2003 jun 19]; 19(2). Disponível em: URL: http:// www.scielo.br

2. Bion FM, Pessoa DCN, Antunes NLM, Medeiros MC. Consumo alimentar e características sócio-econômicas de alunos da Universidade Aberta para a Terceira Idade (UnATI-UFPE). Nutrição Brasil 2003; 2(2):76-82.

3. Bouchard C. Atividade física e obesidade. São Paulo: Manole, 2003. 469 p.

4. Brandão AP, Brandão A, Freitas EV, Campos ME, Pozzan R. Hipertensão arterial no idoso. In: Freitas E, et al organizador. Tratado de Geriatria e Gerontologia. Rio de Janeiro: Guanabara Koogan; 2002. p. 250 - 62.

5. Cabrera MAS. Abordagem da obesidade em pacientes idosos. In: Jacob Filho W, organizador. Promoção da saúde do idoso. São Paulo: Lemos Editorial; 1998. p. 93-108.

6. Cabrera MAS, Jacob Filho W. Obesidade em idosos; prevalência, distribuição e associação com hábitos e co-morbidades. Arq Bras Endocrinol Metabol 2001 [acesso 2003 jun 19]; 45(5). Disponível em: URL: http:// www.scielo.br

7. Consenso brasileiro sobre dislipidemias: detecção, avaliação e tratamento. Arq Bras Cardiol 1996; 67(2):103-28.

8. Dórea EL, Lotufo PA. Framinghan Heart Study e a teoria do contínuo de Pickering: duas contribuições da epidemiologia para a associação entre pressão arterial e doença cardiovascular. Revista brasileira de hipertensabo 2001; 8(2):195 - 200.

9. Frank AA. Estudo antropométrico e dietético de idosos. [mestrado]. Rio de 
Janeiro: Universidade Federal do Rio de Janeiro, Instituto de Nutrição; 1996.

10. Harris MI. Impaired glucose tolerance in the US population. Diabetes Care 1999; 12(2): $464-74$.

11. Hayflick L. Como e por que envelhecemos. 2. ed. Rio de Janeiro: Campus; 1997. 336p.

12. Lerman GI, et al. The prevalence of obesity and its determinants in urban and rural aging mexican. Obes Res 1999: 7(4): 402 - 6.

13. Lin CC, et al. Epidemiology of obesity in elderly people. Yale J Biol Med 1999; 72(6): $385-91$.

14. Lottenberg SA. Obesidade e dislipidemias. In: Harpen A, et al, organizador, Obesidade. São Paulo: Lemos Editorial, 1998. p.145 - 51.

15. Marques APO, Arruda IKG, Espírito Santo ACG, Guerra MD. Consumo alimentar em mulheres com sobrepeso. Textos sobre Envelhecimento 2005; 8(2): 169-86.

16. Marques APO, Arruda IKG, Espírito Santo ACG, Raposo MCF, Guerra MD, Sales TF. Prevalência de obesidade e fatores associados em mulheres idosas. Arq Bras Endocrinol Metabol 2005; 49(3): 441-8.

17. Marucci MFN. Aspectos nutricionais e hábitos alimentares de idosos matriculados em ambulatório geriátrico. [tese]. São Paulo: Universidade de São Paulo, Faculdade de Saúde Pública; 1992.

18. Masaki $\mathrm{KH}$, et al. Association of body mass index with blood pressure in elderly Japanese American men. The Honolulu Heart Program. Hypertension 1997; 29(2): 673 - 7.

19. Monteiro CA, Mondini L, Costa R L. Mudanças na composição e adequação da dieta familiar nas áreas metropolitanas do Brasil (1988-1996). Rev Saúde Pública 2000; 34(3): 251- 8 .

20. Monteiro CJ. Obesidade: diagnóstico, métodos e fundamentos. In: Harpen A, et al, organizador. Obesidade. São Paulo: Lemos Editorial, 1998. p.31-53.

21. Moriguchi EH, Michelon E, Vieira JLC. Dislipidemia em idosos In: Freitas E, et al, organizador. Tratado de Geriatria e Gerontologia. Rio de Janeiro: Guanabara Koogan; 2002. p.239 - 48.

22. Moriguti JC, et al. Nutrição no idoso. In: Oliveira JE, Marchine JS. Ciências nutricionais. São Paulo: Savier;1998. p.239-51.

23. Mykkänen L, Laakso M, Pyörälä K. Association of obesity and distribution with glucose tolerance and cardiovascular risk factors in the elderly. International Journal of Obesity and Metabolic Disorders 1992; 6(9): 695-704.

24. Najas MS, et al. Padrão alimentar de idosos de diferentes estratos socioeconômicos residentes em localidade urbana da Região Sudeste, Brasil. Rev Saúde Pública 1994 [acesso 2003 ago 11]; 28(3). Disponível em: URL: http://www.scielo.br

25. Nasri F. Diabetes Mellitus no idoso.In: : Freitas E, et al, organizador. Tratado de Geriatria e Gerontologia. Rio de Janeiro: Guanabara Koogan; 2002. p.496-501.

26. Papaleo Netto M. O estudo da velhice no século XX: histórico, definição do campo e termos básicos. In: Freitas E, et al, organizador. Tratado de Geriatria e Gerontologia. Rio de Janeiro: Guanbara Koogan; 2002. p.2-12.

27. Popkin BM. The nutrition transition and obesity in the developing world. J Nutr 2001; 131(3):871- 73 .

28. Ramos LR. Epidemiologia do envelhecimento. In: Freitas E, et al, organizador. Tratado de Geriatria e Gerontologia. Rio de Janeiro: Guanabara Koogan; 2002. p.72 - 8.

29. __ Fatores determinantes do envelhecimento saudável em idosos residentes 
em centro urbano: Projeto Epidoso. Cad

Saúde Pública 2003; 19(3): 793-98.

30. Sartorelli DS, Franco S. Tendências do diabetes mellitus no Brasil: o papel da transição nutricional. Cad Saúde Pública 2003; 19(1):S29 - S36.

31. Schieri R. Epidemiologia da obesidade. Rio de Janeiro: EDUERJ; 1998. 140p.

32. Sena JEA. Composição corporal e qualidade de vida em idosos de João Pessoa - PB. [mestrado]. João Pessoa: Universidade Estadual da Paraíba, Programa Interdisciplinar em Saúde Coletiva; 2002.

33. Tavares EL, Anjos LA. Perfil antropométrico da população brasileira. Resultados da
Pesquisa Nacional sobre Saúde e Nutrição. Cad Saúde Pública 1999 [acesso 2002 nov 21];15(4). Disponível em: URL: http:/ / www.scielo.br

34. WHO. Obesity: preventing and managing the global epidemic. Report of a WHO Consultation Group on Obesity. Geneva; 1998.

35. WHO. Physical status: the use and interpretation of anthropometry. Geneva, 1995. (Technical Reports Series, 854).

Recebido em: 19/5/2006

Revisado: 22/11/2006

Aceito: 15/12/2006 